\title{
Analysis of magnetic force production in slider actuators combining analytical and finite element methods
}

\author{
Antônio Flavio Licarião Nogueira \\ Departamento de Engenharia Elétrica \\ Universidade do Estado de Santa Catarina \\ Joinville, Santa Catarina, Brazil \\ E-mail: antonioflavio@ieee.org
}

\begin{abstract}
The production of magnetic forces in a magnetic actuator is the focus of the present analysis. Magnetic circuit analysis is used to investigate the direction as well as the expected order of magnitude of the force that attracts the armature into the central portion of a stationary core. A sequence of finite-element based solutions is used to investigate magnetic saturation and to determine the forcedisplacement characteristic. The analytical approach is based on the principle of virtual work and Ampère's law. The force calculations based on numerical field solutions employ the methods of weighted Maxwell stress tensor and the classical virtual work. The more accurate field-derived solutions are used to explain the limitations of the analytical calculations. At the end, an alternative approach to compute the force numerically is proposed. This method is based on the principle of virtual work, but only one field solution is required.
\end{abstract}

Index Terms-Energy storage, finite element methods, magnetic circuits, magnetic forces.

\section{INTRODUCTION}

Calculation of forces and torques from numerical field solutions is often subject to large numerical errors. Any simplifications made during the modeling of the device, and the numerical approximations assumed during the solution of the field equations result in field distributions that are, inherently, approximate. The evaluation of forces and torques invariably involves some kind of numerical differentiation, and this tends to give rise to errors in the computed forces and torques that are greater than the errors in the potential solutions. A factor that adversely affects the accuracy of some force calculation methods is the presence of magnetic saturation, and this should be considered during the choice of the force calculation method.

There is not a single force calculation method able to produce consistent and accurate results across a range of devices; the geometry of the device and its intended application should thus be considered prior to the actual modeling and choice of the force calculation method. An initial inspection of the device's geometry can provide information regarding the nature of the field distribution, and help to identify regions where a greater amount of field energy is stored, as well as regions where the field solution is more likely to be in error. It is usually possible, at an early stage, to investigate the direction of the developed forces and torques, and their likely distributions. The literature documents extensive research into the magnetic forces produced in linear actuators, as well as into the torques produced by electric motors and other rotating devices [1], [2]. Two of the most popular types of actuators have cores in the shape of " $\mathrm{C}$ " and " $\mathrm{E}$ ", and are commonly referred to as $C$-core and $E$-core actuators, respectively. $C$-core actuators have been chosen by Kim, Lowther and Sykulski [3] to evaluate the accuracy of force computation algorithms developed to determine global forces, as well as force distributions over the surface of a magnetized body. Magnetic cores in the shape of "E" have been used by Barre et al [4] in the study of mechanical vibrations and audible noise emitted by industrial inductors. The experiment reported by Lowther and Freeman [5] to introduce force calculation methods involves force measurements and computer-based simulations of an $E I$-core actuator. Many parametric studies of ac contactors consisting of either, EE-cores [6] or EI-cores [7], are easily found in the literature. Elementary discussions on force development at iron - air interfaces appropriate to linear actuators may be found in [8].

The aim of the present analysis is to investigate different features that affect the force production of a particular magnetic actuator. The problem concerns the computation of the global forces acting on a plunger of $16 \mathrm{~mm}$ height and $20 \mathrm{~mm}$ depth being attracted into a $C$-core. The total magnetomotive force is $5.0 \mathrm{kA}$-turns, 
and an outline of the problem geometry is shown in Fig. 1. Only the parts that affect the electromagnetic action of the slider actuator are presented in the illustration.

The type of slider actuator shown in Fig. 1 is less popular, and its analysis is more difficult than that of the actuators containing armatures that are essentially external to the stationary core. In the latter class of actuators, when the armature moves towards the stationary core, the stored magnetic energy in the air gap regions always increases. In the slider actuator, when the plunger moves in towards the coil, the stored energy in the region between the plunger and the coil becomes smaller, whereas the energy stored in the two overlapping gaps increase. A curious feature of this particular actuator is that the useful, net force attracting the plunger is actually produced by the leakage field in the actuator's window rather than the field in the overlapping gaps.

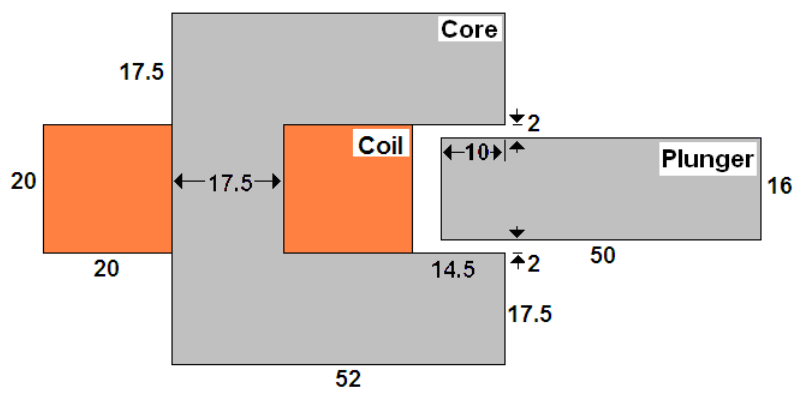

Figure 1. Slider actuator; overlap of 10mm; dimensions in $\mathrm{mm}$

The present study starts with an inspection on the direction and magnitude of the force developed in the slider actuator. This is followed by the planning of the finite element model, especially in its aspects concerning the sequence of static solutions that simulate the displacement of the movable part.

Three force calculation methods are employed in the analysis of the computer-based problems: (i) virtual work; (ii) Maxwell stress tensor; (iii) weighted Maxwell stress tensor. Under their classical formulations, the first two methods can be applied for the force evaluation of practically any device [9]. In the following, the basis of the first two methods are used in the analytical development, whereas the third one is used to compute force values from the finite element solutions. The method of weighted Maxwell stress tensor is recognized for producing accurate and consistent force predictions, and one of its major advantages is its adequacy to the analysis of magnetically saturated devices. The mathematical formulation of this method is presented in [10].

\section{ANALytical CALCUlations}

\section{A. Direction of the force}

Consider a closed surface placed in the air region surrounding the magnetic plunger, as shown in the illustration of Fig. 2. The total magnetic force acting on the body may be found through the integration of the Maxwell field stresses in the air space over this surface [11]. In two dimensions, the surface of integration becomes a contour of integration. In this particular analysis, it is assumed that the magnetic parts are highly permeable. The two short gaps crossed by the segments $a-b$ and $f$ - $g$ are the regions where the $H$-field is more intense, more uniform and where a considerable amount of the system's energy is stored. If each gap is viewed as a magnetic dipole, it becomes clear that attractive forces are developed in both gaps. The attractive force transmitted to the plunger through the face $a-b$ acts in the $+y$ direction, whereas that transmitted through the face $f$ - $g$ acts in the $-y$ direction. Due to the symmetry of the structure, these two forces cancel.

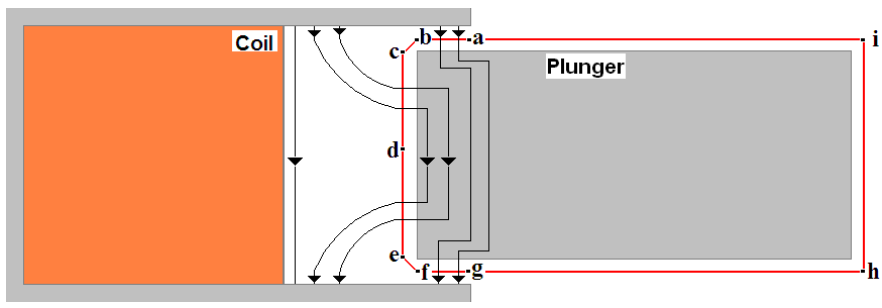

Figure 2. Integration contour and sketch of flux lines

Yet considering the short length of the overlapping gaps, and the resulting uniformity of the $H$-field, it is may be expected a negligible contribution of the fringing fields to the force transmitted through faces $b-c$ and $e-f$. In addition, the faces indicated by $a-i, i-h$ and $h-g$ may be considered remote regions where the $H$-field is also negligible. The force acting on the face $c$-e should then be considered. The field distribution in the actuator's 
window is very difficult to determine analytically, although not numerically. With the aid of the idealized flux lines that appear in the illustration, it is possible to observe that part of the magnetic flux emerging from the upper limb of the stationary core bends towards the front edge of the plunger and strikes the air-iron interface at right angles. On the left hand side, close to the coil, a smaller proportion of the magnetic flux crosses the air region in the $-y$ direction, but gives no contribution to the developed force. As a result of the attractive force represented by the bending flux lines that cross the faces $c$ - $d$ and $d-e$, and observing the symmetry of the structure with respect to a horizontal line that bisects the device, it becomes clear that the vertical components of the forces pulling the upper and lower halves of the front edge in opposite directions, cancel. Therefore, the net force that tends to move the magnetic plunger is wholly accounted for by the horizontal components of the force density in the $-x$ direction. In other words, the net force can be simply calculated by numerical integration of the force densities along the faces $c-d$ and $d-e$.

\section{B. Magnitude of the force}

To estimate the order of magnitude of the force by the method of virtual work, it is assumed magnetic linearity in a way that the system's coenergy can be approximated by its stored magnetic energy. In addition, it is assumed the presence of uniformly developed magnetic fields in two adjacent air-filled regions, namely the actuator's window and the overlapping gaps. The essence of this simplified approach is the assumption that the change in the system's energy with movement is mainly confined to these air regions that are "deformed" by the movement.

A cross-sectional view of the device is shown in Fig. 3. The height $l_{w}$ of the window is $20 \mathrm{~mm}$, and the length $l_{g}$ of each overlapping gap is $2 \mathrm{~mm}$. The depth of the device is $z=20 \mathrm{~mm}$, and all parts of the actuator are considered longitudinally uniform. In this illustration, $a$ is a constant and denotes the maximum distance the plunger can move into the cavity; the variable $x$ is measured from left to right and represents the distance between the coil's right edge and the plunger's front edge. According to this, the amount of overlap is $d=a-x$. In the following development, $\delta x$ denotes the positional displacement associated with the classical virtual work method. The selection of the positional displacement defines the sequence of perturbed system solutions used to obtain, either a single force estimate or a force-displacement characteristic. In the illustration of Fig. 3, the variable $\delta x$ may be interpreted as the small amount of "deformation" in the air-filled regions caused by the plunger movement in the $-x$ direction.

The derivation assumes no change in the mean value of the flux density $B$ of the air-filled regions at two consecutive positions. Therefore, the density of magnetically stored energy does not change with movement, and the change in stored energy is expressed in terms of the volumes of air of each adjacent region, before and after the displacement.

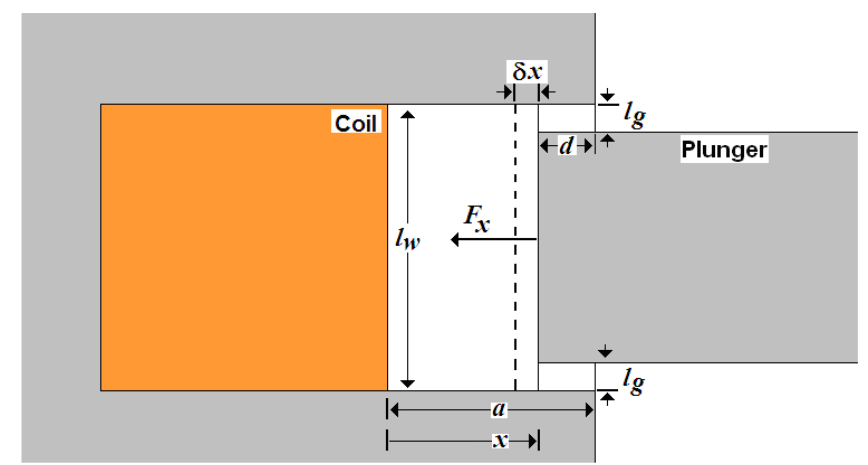

Figure 3. Adjacent volumes of air deformed by the movement of the plunger

Let $E_{w}$ denote the magnetic stored energy in the volume of air, $V_{w}$, of the stator window. For a mean flux density $B_{w}$ in the window region, the density of magnetic stored energy is $\left(B_{w}\right)^{2} / 2 \mu_{0}$, and $\mu_{0}=4 \pi \times 10^{-7} \mathrm{H} / \mathrm{m}$ is the magnetic permeability of free space. The corresponding magnetic stored energy is

$$
E_{w}=\frac{\left(B_{w}\right)^{2}}{2 \mu_{0}} V_{w} .
$$

Similarly, let $E_{o}$ denote the magnetic stored energy in the volume of air, $V_{o}$, of one overlapping gap. For a mean flux density $B_{o}$ in the gap region, the density of magnetic stored energy is $\left(B_{o}\right)^{2} / 2 \mu_{0}$. The corresponding stored energy is 


$$
E_{o}=\frac{\left(B_{o}\right)^{2}}{2 \mu_{0}} V_{o}
$$

After a short displacement $\delta x$ of the plunger in the $-x$ direction, the width of the window decreases from $x$ to $(x-\delta x)$, and its volume decreases from $x l_{w} z$ to $(x-\delta x) l_{w} z$. The width of the overlapping gap increases from $d$ to $(d+\delta x)$, and its volume increases from $d l_{g} z$ to $(d+\delta x) l_{g} z$.

The energy difference $\delta E$ is calculated taking into account the reduction in the volume $V_{w}$ of the window, and the simultaneous increase in the volume of the two overlapping gaps. After some algebraic manipulations, one gets

$$
\delta E=2 \frac{\left(B_{o}\right)^{2}}{2 \mu_{0}}\left(\delta x l_{g} z\right)-\frac{\left(B_{w}\right)^{2}}{2 \mu_{0}}\left(\delta x l_{w} z\right)
$$

According to the principle of virtual work, an estimate for the force, $F_{x}$, can be obtained by the ratio of the energy difference $\delta E$ with respect to the small positional displacement $\delta x$,

$$
F_{x}=\frac{\delta E}{\delta x}=2 \frac{\left(B_{o}\right)^{2}}{2 \mu_{0}}\left(l_{g} z\right)-\frac{\left(B_{w}\right)^{2}}{2 \mu_{0}}\left(l_{w} z\right)
$$

The closed contours $C_{1}$ and $C_{2}$ that appear in the illustration of Fig. 4 are used in the calculation of the magnetic flux densities in the actuator's window and overlapping gaps. If the magnetic field intensity $H$ is assumed to be negligible in the magnetized parts of the contours, application of Ampère's law along the contour $C_{l}$ gives an estimation for the flux density $B_{w}$ in the actuator's window,

$$
B_{w}=\frac{\mu_{0} I}{l_{w}}=0.314 T
$$

In a similar way, integration along the contour $C_{2}$ gives an estimation for the flux density $B_{o}$ in each of the two gaps,

$$
B_{o}=\frac{\mu_{0} I}{2 l_{g}}=1.571 T
$$

An estimate for the order of magnitude of the force can now be obtained. Based on the flux densities computed by (5) and (6), the magnitude of the force calculated by (4) is $62.8 \mathrm{~N}$. In a later section, this force value will be contrasted with the force-displacement characteristic.

In practical terms, the accuracy of this simplified approach is mainly related to the geometrical dimensions of the actuator, to know: i) the distance $a$ between the coil's right edge and the rightmost edge of the $C$-core; ii) the width $d$ of the overlapping gaps, as indicated in Fig. 3. For larger widths, the approximations to the distributions of flux density given by (5) and (6) become less accurate. This loss of accuracy clearly affects the calculation of parameters like the forces calculated by (4). Fortunately, most magnetic actuators produce motion over a limited range, and the method gives results of sufficient accuracy for many practical purposes.

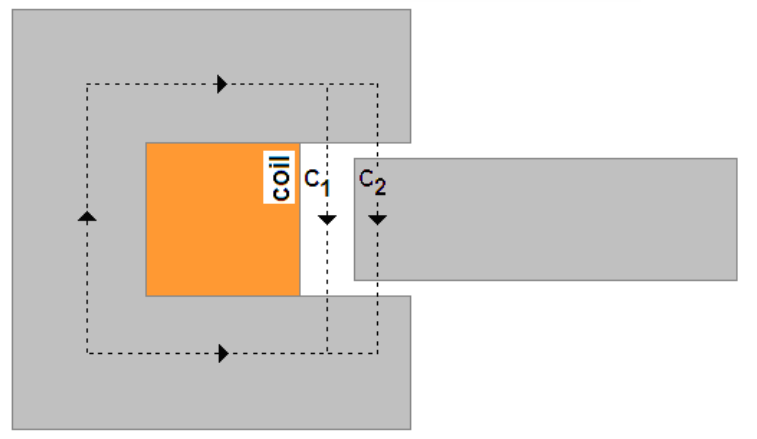

Figure 4. Contours for the application of Ampère's law 


\section{FINITE ELEMENT MODEL}

Numerical field solutions have been obtained using the two-dimensional Cartesian magnetostatic processors and solver of the simulation software FEMM [12]. To permit two-dimensional analysis, the actuator is considered longitudinally uniform and long enough so that end effects are negligible. The solutions are obtained for the magnetic vector potential $\boldsymbol{A}$ which, in a two-dimensional analysis, is assumed entirely $z$ directed. It is then possible to work simply with a scalar distribution $A$ which denotes the longitudinal component of the vector $\boldsymbol{A}$. The nonlinear magnetization characteristic of the $M-36$ steel represents the behavior of the material that forms the magnetic parts. First-order triangular finite elements are employed in all computations. The boundary conditions are homogeneous Dirichlet; the condition $A=0$ is applied to a remote circular boundary whose radius is $40 \mathrm{~cm}$ and represents approximately 6 times the leading dimension of the device [13].

\section{A. Simulation of movement}

A sequence of static solutions is used to obtain the force-displacement characteristic of the slider actuator. In this sequence, the plunger moves $11 \mathrm{~mm}$ in the $-x$ direction. The initial position represents an overlap of $1 \mathrm{~mm}$, and the final position represents an overlap of $12 \mathrm{~mm}$. The plunger movement is simulated using the artifice of material re-identification to change materials and their properties in problems representing successive positions [14].

\section{RESULTS}

\section{A. Flux densities}

The flux plot presented in Fig. 5 is used to illustrate the general pattern of the solutions. The plot refers to the configuration of the slider actuator where the overlapping gaps are $4 \mathrm{~mm}$ wide. At this position, the energy storage in the two overlapping gaps is approximately $1 \%$ larger than that in the window region. With the aid of this plot it can be observed that the field in the two overlapping gaps is essentially uniform, and this is mainly due to the short length of these gaps, namely $2 \mathrm{~mm}$. There is also a component of the total flux that avoids the overlapping gaps and crosses the magnetic plunger. This leakage field is completely symmetrical with respect to a horizontal line that bisects the device and does not contribute to the force production.

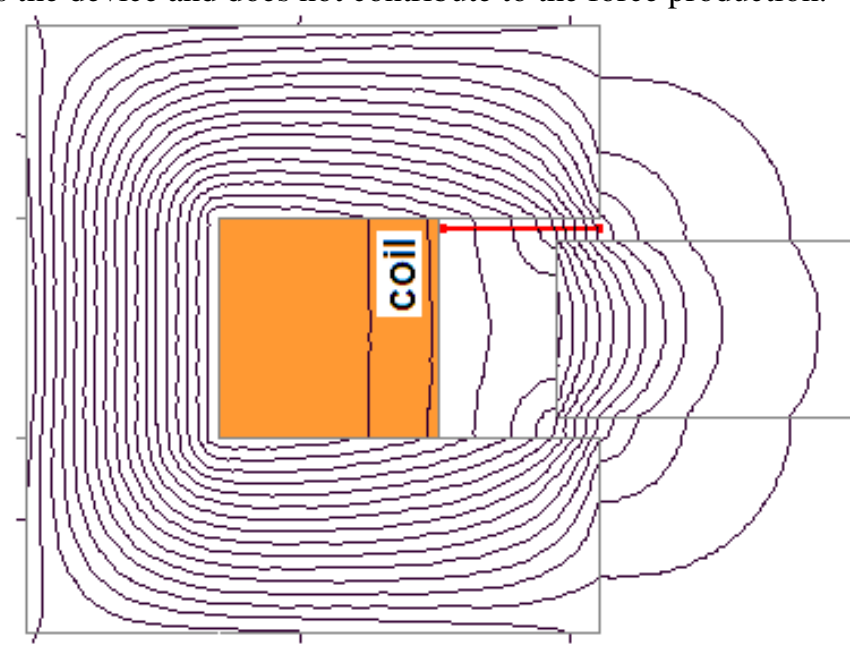

Figure 5. Flux lines and contours of inspection for an overlap of $4 \mathrm{~mm}$

The plot reveals that, in the region of the actuator's window, there is no uniform developed field. The distribution of these flux lines is in accordance with the theoretical predictions made in the earlier analytical development. On the left hand side of the window, the magnetic flux crosses the space between the upper and lower limbs more or less vertically. Close to the front edge of the plunger one can observe an increasing concentration and inhomogeneity of the field, especially near the upper and lower corners of the plunger; close to the tips, flux lines bend towards the nearer side of the stationary core with a relatively small radius of curvature. The main contribution to the net force is due to the stresses in these regions.

Inspection of the numerical values of flux densities is made with the aid of the horizontal contour that appears in Fig. 5, in red color. This contour is defined from left to right, starts very close to the coil, and crosses the window and the upper gap. The variations of flux densities along this contour are presented in the graph of Fig. 6. The curve in blue color represents the flux distribution in the upper sector of the window's region. This is followed by the curve in red color that represents the flux distribution in the middle of the upper gap. Both 
curves have been obtained from the potential solution that represents overlapping gaps of $4 \mathrm{~mm}$ width. The solid horizontal line represents the estimate $B_{w}=0.314 \mathrm{~T}$, calculated by (5) for the flux density in the window's region. The dashed horizontal line represents the estimate $B_{o}=1.571 \mathrm{~T}$, calculated by (6) for the flux density in the overlapping gaps.

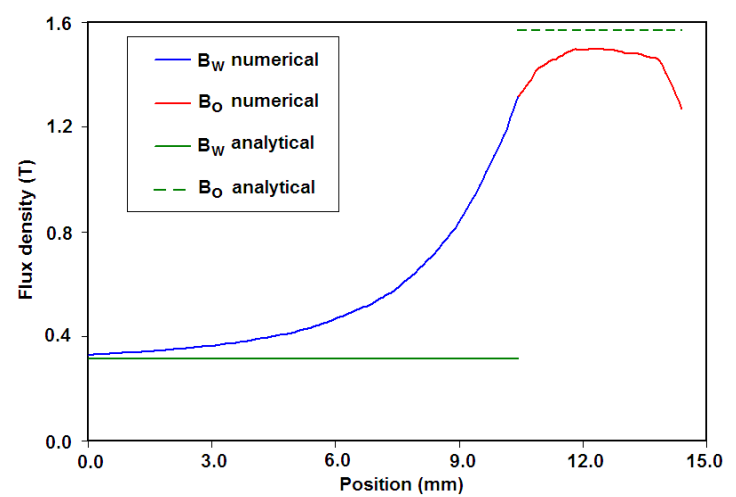

Figure 6. Magnitude of flux densities in the window and upper gap

Observation of the characteristics presented in Fig. 6 confirms the earlier predictions related to the general pattern of the field distributions, i.e. the uniformity of the field in the short gaps and the inhomogeneity of the field in the upper portion of window's region.

The analytical calculations based on Ampère's law ought to be compared with the more precise values obtained from the finite element solution. In the window's region, the analytical estimate is $B_{w}=0.314 \mathrm{~T}$. This value is clearly underestimated, and represents only $58 \%$ of the mean value of the curve shown in blue color, namely $0.538 \mathrm{~T}$. In the overlapping gap, the discrepancy between the analytical and numerical estimates is not so severe. The analytical estimate is $B_{o}=1.571 \mathrm{~T}$, and this value is only $8.6 \%$ larger than the mean value of the curve shown in red color, namely $1.447 \mathrm{~T}$.

\section{B. Stored energy and saturation}

Variations of the magnetically stored energy in the window's region and overlapping gaps with the respect to the amount of overlap are presented in the graph of Fig. 7.

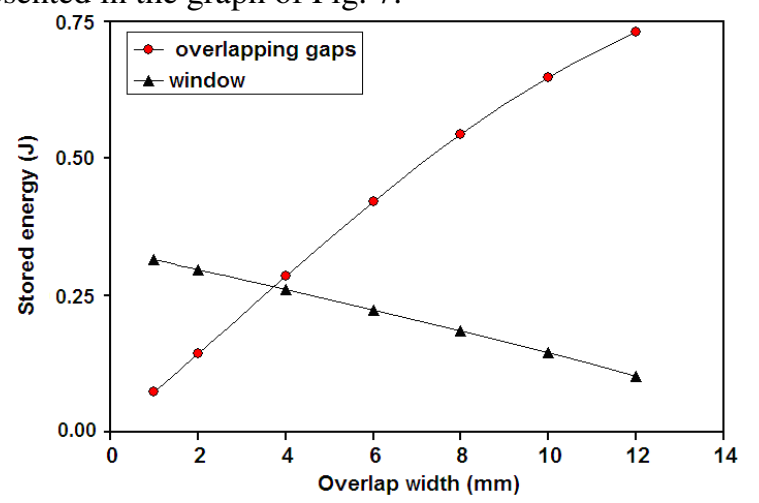

Figure 7. Variation of stored energies with respect to the width of overlap

Observation of the two characteristics that appear in Fig. 7 reveals that when the plunger moves in the $-x$ direction, the amount of energy stored in the window's region decreases. Simultaneously, it can be observed a much more pronounced increase in the amount of energy stored in the two air gaps. The energy stored in the coil's region decreases only $3.6 \%$ over the simulated excursion, and the change in the energy stored in the external leakage fields is negligible.

Inspection of magnetic saturation has been made by comparing the values of stored energy and coenergy. The difference in the values of energy and coenergy indicates the presence of magnetic saturation in the magnetic parts of the actuator. Presented results show that saturation effects only become significant for larger overlaps; when the overlap increases from 10 to $12 \mathrm{~mm}$, the relationship between the values of coenergy and energy rises rapidly from 11 to $15.4 \%$. When saturation is present, a considerable number of force calculation methods must be disregarded, and these include, e.g. some linearized approaches based on the virtual work principle [15]. This is not the case of the method employed here to obtain the force-displacement characteristic, i.e. the weighted Maxwell stress tensor method [10]. This method has proved to be adequate for extracting accurate and 
consistent force predictions from imperfect numerical field solutions, and its formulation does not include any simplifications regarding magnetic linearity.

\section{Force calculations}

The variation of the force along the simulated excursion is shown in the graph of Fig. 8. The numerical force estimates have been obtained from the finite element solutions employing the methods of weighted Maxwell stress tensor and the classical virtual work.

The single force estimate calculated analytically by (4) is $62.8 \mathrm{~N}$. In fact, force values vary substantially along the simulated excursion, as shown in the graph of Fig. 8. However, all that is expected from the simplified analytical approach is an estimate for the order of magnitude, and this estimate may be considered satisfactory for many practical purposes. When compared with the predictions by the weighted tensor method, the numerical error is $4.5 \%$ when compared to the peak force, and $9.8 \%$ when compared to $57.19 \mathrm{~N}$, the mean value of the force-displacement characteristic.

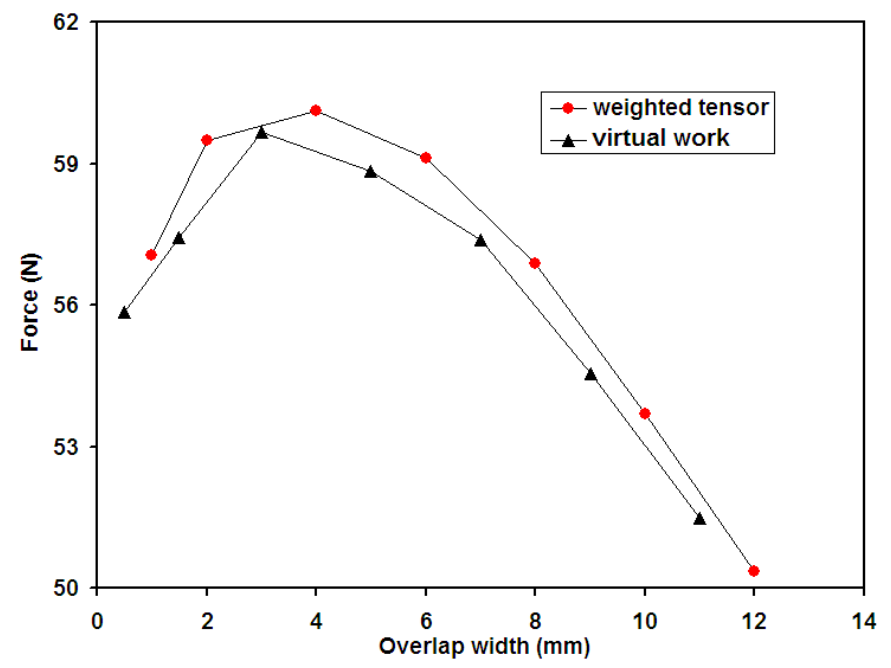

Figure 8. Variation of attractive force with respect to the width of overlap

A close observation of the characteristic marked with circles shows that, when the overlap width is 4 mm the force reaches its maximum value, namely $60.12 \mathrm{~N}$. This maximum value occurs at the plunger's position where the amount of energy stored in the force producing region has just become smaller than that stored in the small gaps. This helps to explain why further increases in the overlap width actually lead to a reduction in the developed force. In other words, for overlaps greater than $4 \mathrm{~mm}$, a greater proportion of the system's energy will be stored in the air-filled regions that give no contribution to the production of force.

\section{Alternative Force CALCUlation APPROACH}

The force production in the actuator is intimately related to the change in the magnetic energy stored in the non-magnetic parts of the device. The total energy $E_{t}$ of the actuator has three major components: i) a constant component $E_{c}$ associated with the coil; ii) a component $E_{w}$ associated with the window's region; and a component $E_{o}$ associated with the two overlapping gaps. In this way, the force can be calculated only in terms of the variation of the energies $E_{w}$ and $E_{o}$ with respect to displacement.

According to the illustration of Fig. 3, the variable $x$ denotes the width of the actuator's window, whereas the width of the overlapping gaps is $(a-x)$. The attractive force can be expressed as

$$
F=\frac{E_{o}}{(a-x)}-\frac{E_{w}}{x}
$$

where $a$ is a constant and denotes the maximum distance the plunger can move into the cavity.

Each of the two energies $E_{w}$ and $E_{o}$ represents a fraction of the total energy $E_{t}$. Each fraction can be determined as the ratio of the flux crossing the region with respect to the total flux.

For the field solution where the amount of overlap is $4 \mathrm{~mm}$, the total flux $\phi_{t}$ is $0.536 \mathrm{mWb}$. The flux crossing the window's region is $0.221 \mathrm{mWb}$ and represents a fraction $f_{w}=0.2094$. The flux crossing the overlapping gaps is $0.123 \mathrm{mWb}$ and represents a fraction $f_{o}=0.2298$.

The total stored energy is $1.550 \mathrm{~J}$. The energy stored in the window's region can be computed as 


$$
E_{w}=f_{w} E_{t}=0.1754 J
$$

The energy stored in the overlapping gaps is

$$
E_{o}=f_{o} E_{t}=0.3561 \mathrm{~J}
$$

The magnitude of the force computed from (7) is $60.80 \mathrm{~N}$. This value compares very well with that calculated by the weighted tensor method, to know $60.12 \mathrm{~N}$. It is worth noting that the latter calculation has been performed in a completely different way. This excellent agreement between magnetic force predictions by different approaches suggests consistent and completely satisfactory force estimates for most practical purposes.

\section{CONCLUSIONS}

The device under analysis is a type of slider actuator containing a movable plunger that is attracted into the interior region of a stationary $C$-core. The discussion shows one way to investigate the direction of the developed force using a surface-integration method. A simplified analytical approach that combines the principle of virtual work and Ampère's law is used to investigate the magnitude of the force. This approach represents a useful method to figure out the order of magnitude of developed forces and can be applied quickly and easily. The artifice of material re-identification has allowed the use of a single finite element model to simulate the plunger's movement. The field-derived distributions of flux densities and stored energies in different regions show good agreement with the theoretical predictions made in the initial analytical development. The force-displacement characteristic is consistent with the physical understanding of the device in its aspects related to energy storage and force production. The study of force production based on the proportions of magnetic energy stored in different regions of the device brings considerable insight into the subject, and should be of value to people working with electromagnetic engineering education, as well as to designers working with device's performance prediction.

\section{REFERENCES}

[1] A.F. Licariao-Nogueira, Computation of cogging torques in permanent-magnet machines using the finite element method, PhD dissertation, University of Wales, Cardiff, 1992

[2] D. Zhu, S. Roberts, M.J. Tudor and S.P. Beeby, "Design and experimental characterization of a tunable vibration-based electromagnetic micro-generator", Sensors and Actuators A: Physical, vol. 158, no. 2, pp. 284-293, 2010.

[3] D.H. Kim, D.A. Lowther and J.K. Sykulski, "Efficient force calculations based on continuum sensitivity analysis", IEEE Transactions on Magnetics, vol. 41, no. 5, pp. 1404-1407, 2005.

[4] O. Barre, B. Napame, M. Hecquet and P. Brochet, "Acoustic noise emitted by passive components in magnetic devices and design of a low-noise industrial inductor", COMPEL, vol. 27, no. 5, p. 1053, 2008.

[5] D.A. Lowther and E.M. Freeman, "A new approach to using simulation software in the electromagnetics curriculum", IEEE Transactions on Education, vol. 36, no. 2, pp. 219-222, 1993.

[6] J.D.R. Ruiz and A.G. Espinosa, "A novel parametric model for ac contactors", IEEE Trans. Magn., vol. 44, no. 9, pp. 2215-2218, 2008.

[7] F. Marion and P. Meneroud, "Optimisation of a contactor dimensions", Flux Magazine, no. 45, pp. 12-13, May 2004.

[8] C.B. Gray, Electrical machines and drive systems, Longman Singapore Publishers (Pte) Ltd, 1989, pp. 74-81.

[9] A.F.L. Nogueira, "Cálculo de forças eletromecânicas pelos métodos do trabalho virtual e tensor de Maxwell" Available: http://www.joinville.udesc.br/portal/professores/nogueira/materiais/

[10] S. McFee, J.P. Webb and D.A. Lowther, "A tunable volume integration formulation for force calculation in finite-element based computational magnetostatics", IEEE Trans. Magn., vol. 24, no. 1, pp. 439-442, 1988.

[11] J.C. Maxwell, Treatise on electricity and magnetism, Clarendon Press, 1904, vol. I, Ch. 5 and vol. II, Ch. 11.

[12] D. Meeker, Finite element method magnetics, user's manual, 2010.

[13] A.F.L. Nogueira, "A case study on open boundary techniques for electromagnetic field problems with translational symmetry", Journal of Microwaves, Optoelectronics and Electromagnetic Applications, vol. 9, no. 1, June 2010.

[14] D.A. Lowther and P.P. Silvester, Computer-aided design in magnetics, Springer-Verlag, New York, $1986, \mathrm{p} .195$.

[15] A.F. Licarião Nogueira, "Computation of forces using mean and difference potentials", in Proc. of the 17 Conference on the Computation of Electromagnetic Fields, Florianópolis, Brazil, 2009. 